\title{
Efeito da manipulação visceral na dor, mobilidade e incapacidade funcional em pessoas com capsulite adesiva de ombro direito
}

\author{
Effect of visceral manipulation on pain, mobility \\ and functional disability in subjects with right \\ shoulder Adhesive Capsulitis
}

\author{
Aarti Ghillodia' 1 (1) \\ Balaji Karunanithi Gandhi ${ }^{2}$ (1)
}

'Autor para correspondência. Chitkara University (Rajpura). Punjab, India. aarti290894@gmail.com ${ }^{2}$ Chitkara University (Rajpura). Punjab, India. balaji.gandhi@chitkara.edu.in

RESUMO | INTRODUÇãO: A capsulite adesiva é uma condição dolorosa comum e debilitante da articulação glenohumeral que afeta cerca de 2 a $5 \%$ da população. Os órgãos internos e o sistema musculoesquelético são interligados por meio das membranas de sustentação. A tensão nessas membranas pode causar ainda mais restrições mecânicas, dor nas estruturas musculoesqueléticas e também restringir a mobilidade de órgãos viscerais. OBJETIVO: Explorar o efeito da manipulação visceral específica de órgãos na capsulite adesiva em pessoas afetadas no ombro direito. METODOLOGIA: Vinte indivíduos com capsulite adesiva foram avaliados para elegibilidade, dos quais 14 foram recrutados usando o método de amostragem por conveniência. Duas sessões de manipulação visceral comparada com manipulação placebo foram administradas nas primeiras duas semanas, seguidas de duas sessões de manipulação visceral do fígado nas duas semanas seguintes. $O$ goniômetro digital, o nível de dor, a incapacidade no ombro e o esfigmomanômetro modificado foram usados para medir os resultados pré e pós intervenção. RESULTADOS: A comparação dos resultados entre os grupos mostrou uma diferença estatisticamente significativa nos escores de amplitude de movimentos [flexão $(p=0,001)$, rotação externa $(p=0,001)$, rotação interna $(p=0,001)]$, dor no ombro e escore do índice de incapacidade $(0,004)$. Nenhuma mudança significativa foi observada no resultado de força. CONCLUSÃo: Duas sessões de manipulação visceral para o fígado podem ser benéficas na melhora da mobilidade do ombro direito, dor e incapacidade.

O número de registro no Clinical Trial é 019/09/028271.

PALAVRAS-CHAVE: Capsulite adesiva. Manipulação visceral. Mobilidade. Dor. Fígado.
ABSTRACT | INTRODUCTION: Adhesive capsulitis is a common, painful and debilitating condition of the glenohumeral joint affecting about $2-5 \%$ of the population. Internal organs and musculoskeletal system are inter-connected through the support membranes. Tension in these support membranes may further cause mechanical restrictions and pain in musculoskeletal structures and also restrict the mobility of the visceral organ. OBJECTIVE: To explore the effect of organ specific visceral manipulation on adhesive capsulitis in subjects with right shoulder adhesive capsulitis. METHODOLOGY: Twenty subjects with adhesive capsulitis were assessed for eligibility, out of which fourteen subjects were recruited using convenience sampling method. Two sessions of Placebo Visceral Manipulation was administered for the first two weeks, followed by a two sessions of visceral manipulation of liver for next two weeks. Digital goniometer, Shoulder pain and disability index and Modified sphygmomanometer were used to measure the pre and post outcomes. RESULTS: Between group comparison of the outcomes showed a statistically significant difference in the shoulder range of motions scores [flexion $(p=0.001)$, external rotation $(p=0.001)$ and internal rotation $(p=0.001)$ ] and Shoulder pain and disability index score (0.004). No significant changes were observed in the strength outcome. CONCLUSION: Two sessions of visceral manipulation for the liver may be beneficial in improving the right shoulder mobility, pain and disability.

Clinical Trials number (REF/2019/09/028271 N)

KEYWORDS: Adhesive Capsulitis. Visceral Manipulation. Mobility. Pain. Liver. 


\section{Introdução}

A capsulite adesiva (CA) ou "ombro congelado" é uma condição patológica incapacitante da articulação do ombro, caracterizada por rigidez, dor e disfunção. O consenso americano de cirurgiões de ombro e cotovelo define capsulite adesiva como "restrição fisiológica ativa e passiva do movimento do ombro para a qual radiografias da glenohumeral são essencialmente normais| ${ }^{1}$. Acredita-se que a inflamação no espaço dos músculos rotadores resulta em movimento doloroso, leva à subsequente fibrose e rigidez que limita os movimentos?2.

A CA comumente se apresenta na sexta década de vida, com pico de idade aos 56 anos, e é mais comum em mulheres do que em homens ${ }^{3}$. De 3 a $5 \%$ dos casos de ombro congelado são relatados na população em geral e até $20 \%$ nas pessoas com diabetes. $\mathrm{Na}$ capsulite adesiva primária, o envolvimento bilateral do ombro é relatado em $40 \%$ a $50 \%$ dos casos e, em alguns casos, a extremidade não dominante é mais comumente envolvida 4 .

A manipulação visceral (MV) foi desenvolvida pelo osteopata francês Jean-Pierre Barral. É uma mobilização fascial de um órgão baseada na livre movimentação do corpo. Qualquer restrição nos tecidos e órgãos podem levar à disfunção vascular, neural, musculoesquelética ou pulmonar ${ }^{6}$. Portanto, as restrições viscerais levam ao aumento das restrições musculares e aliviam a intensidade da dor nos tecidos supridos pelo nível espinal relativo por meio dos reflexos risomáticos viscerais. Devido a isso, as fibras aferentes afetam o nervo motor ou simpático ${ }^{5}$.

A MV avalia e trata a disfunção estrutural e fascial que leva ao aumento da comunicação proprioceptiva dentro do corpo e ao alívio dos sintomas de dor e disfunção. De acordo com a hipótese deste estudo, a MV visa restaurar a mobilidade do fígado por aliviar as restrições nos três planos de movimento, o que leva à melhora dos sintomas do ombro direito (dor e mobilidade). Há uma carência de evidências terapêuticas objetivas para apoiar o efeito da manipulação visceral na dor do ombro direito, mobilidade e incapacidade funcional em indivíduos com CA. O objectivo do estudo foi determinar o efeito da manipulação de órgão específico em indivíduos com ombro direito acometido por capsulite adesiva.
Método

\section{Participantes}

Uma amostra de conveniência de 20 indivíduos com diagnóstico de capsulite adesiva do lado direito foram incluídos no estudo. Dos 20 indivíduos selecionados, 14 foram diagnosticados com adesiva capsulite do ombro direito, com restrição de movimento há três meses, com idade entre 45-65 anos, ambos os sexos, recrutados para o estudo por meio de uma amostragem por conveniência. O paciente com história de cirurgia recente de pescoço, trauma no ombro direito, e portadores de doenças hepáticas crônicas foram excluídos do estudo. Este estudo piloto foi conduzido em um departamento de Fisioterapia de um hospital multi-especializado reconhecido, em Mohali. O protocolo do estudo foi aprovado pelo Comité de Ética Institucional do hospital Mohali e registrado no Clinical Trials sob o número 2019/09/028271. O consentimento informado por escrito foi obtido dos participantes antes do estudo.

\section{Medida de desfecho}

As medidas de desfechos primários utilizados foram obtidas pelo índice de inabilidade e dor de ombro (SPADI), dados de goniômetros e esfigmomanômetros digitais que foram usados como medidas de desfecho secundário.

O SPADI é um questionário autorreferido composto por 13 itens em duas subescalas: 5 itens de dor e 8 itens de deficiência, usando uma escala numérica de 11 pontos sobre dificuldade de 0 a 10 . A escala produz uma pontuação total de 130 e é subdividida em 50 pontos para dor e 80 para deficiência. As consistências internas das subescalas do SPADI foram altas $(a>0,92)$. Correlações $r>0,60$ foram observadas entre o SPADI e a dor relatada em uma escala de dor VAS durante o movimento ativo e passivo. A escala demonstrou ser uma ferramenta válida e confiável para medir a dor e a incapacidade em ambientes de atenção primária com boa validade de construto para uma variedade de condições de ombro, incluindo ombro congelado ${ }^{6}$. Um goniômetro digital portátil de bolso e um laser para medir ângulos articulares foram também usados. A confiabilidade do goniômetro digital intra-observadores foi estabelecida entre 0,82 e 0,91 e inter-observadores entre 0,89 a 0,98 . 
O esfigmomanômetro foi usado para medir a força muscular. O método do esfingnomanômetro modificado apresentou excelente confiabilidade teste-reteste (ICC $=0,995-0,999)$ para avaliar a contração isométrica voluntária máxima dos músculos do ombro

\section{Intervenção}

Os indivíduos receberam duas sessões de manipulação visceral com placebo (MVP) por duas semanas. $O$ sujeito do estudo foi posicionado em decúbito dorsal na maca com a região do abdôme exposta. O terapeuta posicionado do lado direito do paciente com a cabeça voltada para a direita, posicionou a mão direita espalmada sobre o abdome do participante na região epigástrica direita ou hepática, pontas dos dedos acima da linha esterno-xifóide, sem movimentação do tecido. A sessão foi dada uma vez por semana durante 10 minutos.

Após as primeiras duas semanas, os indivíduos receberam duas sessões de manipulação visceral (MV) do fígado durante as duas semanas seguintes. As manipulações do fígado envolveram três técnicas em planos diferentes. Cada técnica foi repetida por 3 ciclos em uma sessão, a sessão completa durou 15 minutos, uma vez por semana. Foram utilizadas três técnicas de manipulações do fígado em três planos diferentes, em técnicas de tratamento nos planos coronal, transversal e sagital. As técnicas estão demonstradas nas figuras 1 a 4 .

Técnicas de tratamento da mobilidade no plano transversal - Cada sujeito foi instruído a deitar-se confortavelmente na maca de tratamento, deitado em decúbito lateral, com o lado direito voltado para cima e ambas as pernas dobradas em direção ao peito e o terapeuta posicionou-se do lado direito do paciente. A manipulação do fígado começou com a mão esquerda do terapeuta sobre a região epigástrica direita (entre a quinta e décima costela); e a mão direita atrás da mão esquerda no topo do arco costal direito ventrolateral. O terapeuta posicionou as mãos para carregar a fáscia e conectar o fígado atrás das costelas e seguir o movimento (rolamento medial / lateral) do fígado na direção da facilidade. Este ciclo foi repetido por 3 repetições.

Técnica de tratamento da mobilidade no plano coronal - Cada sujeito foi instruído a deitar-se confortavelmente na maca de tratamento em decúbito dorsal horizontal e o terapeuta posicionou-se do lado direito do paciente em direção à cabeça. O terapeuta posicionou a mão direita (dedos apontando para o umbigo) sobre a quinta / sexta costelas e a mão esquerda abaixo da direita sobre o arco costal direito. O terapeuta colocou as mãos para carregar a fáscia para conectar o fígado atrás da costela e seguir o movimento inferior (em direção à pelve) e superior (em direção ao ombro direito) do fígado na direção da facilidade. Este ciclo foi repetido por 3 repetições.

Técnica de tratamento da mobilidade no plano sagital - O sujeito estava deitado em decúbito lateral com o lado direito para cima e ambas as pernas dobradas em direção ao tórax e o terapeuta atrás do paciente em direção ao lado da cabeça. $\mathrm{O}$ terapeuta colocou sua mão esquerda verticalmente na parte posterior da quinta / sexta costela no arco costal direito e o lado direito verticalmente na região anterior do arco costal direito, com ambos os polegares apontando para o ombro direito. O terapeuta posicionou as mãos para carregar aa fáscias para conectar o fígado e seguir o movimento (rolamento anterior / posterior) do fígado na direção da fluidez. Este ciclo foi repetido por 3 vezes.

Figura 1. Técnica de manipulação placebo

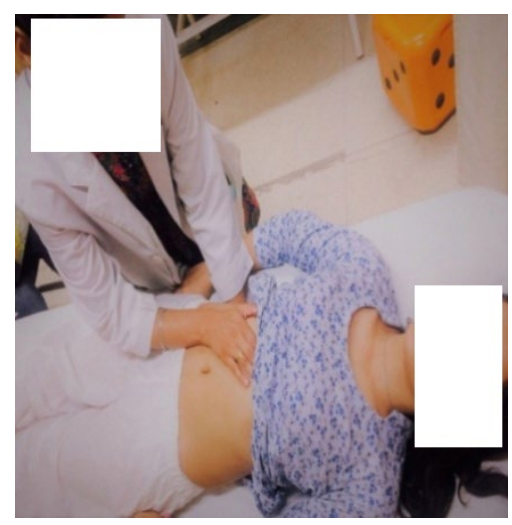


Figura 2. Manipulação visceral no plano frontal

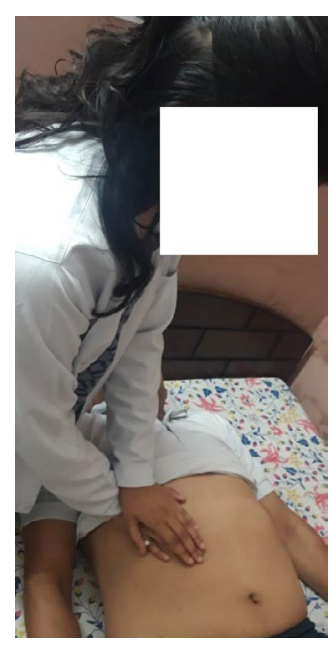

Figura 3. Manipulação visceral no plano transversal

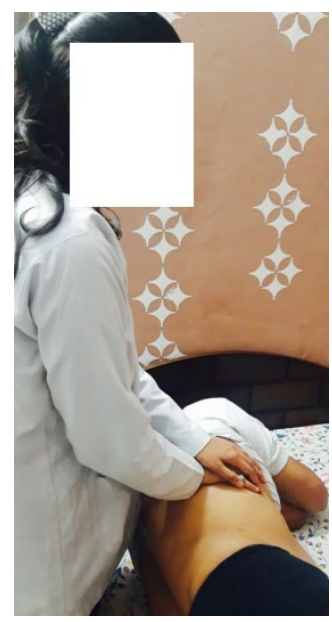

Figura 4. Manipulação visceral no plano sagital

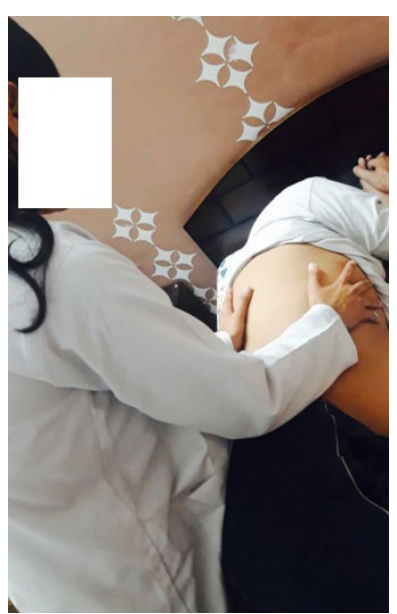




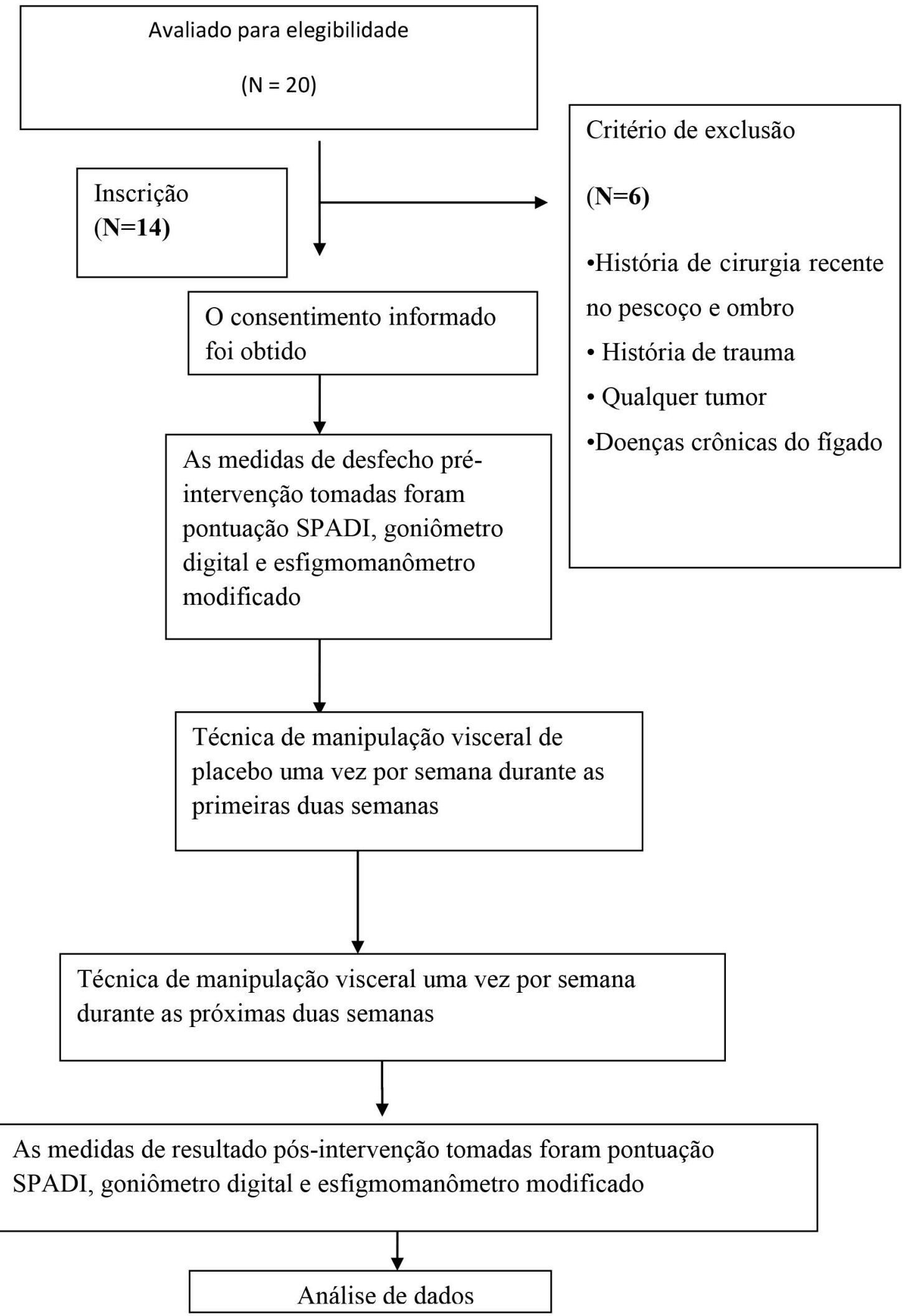




\section{Resultados}

Quatorze indivíduos com uma idade média de 56,5+6,51 anos foram recrutados para este estudo. As características demográficas e clínicas basais são apresentadas na Quadro 1. Entre os sujeitos, 78,5\% eram mulheres, 21,4\% homens, 28,5\% eram diabéticos e 71,4\% não diabéticos.

Quadro 1. Dados demográficos dos sujeitos do estudo

\begin{tabular}{|l|l|l|}
\hline Características dos participantes do estudo $(\mathrm{N}=14)$ & Número de participantes (N) \\
\hline \multirow{4}{*}{ Idade em anos) } & $41-50$ & 5 \\
\cline { 2 - 3 } & $51-60$ & 3 \\
\cline { 2 - 3 } & $61-70$ & 6 \\
\hline \multirow{2}{*}{ Gênero } & Masculino & 3 \\
\cline { 2 - 3 } & Fêmea & 11 \\
\hline \multirow{2}{*}{ Diabetes } & Diabético & 4 \\
\cline { 2 - 3 } & Não diabético & 10 \\
\hline
\end{tabular}

Quadro 2. Mostrando dentro do grupo a comparação de todas as medidas de resultado de ambos os grupos

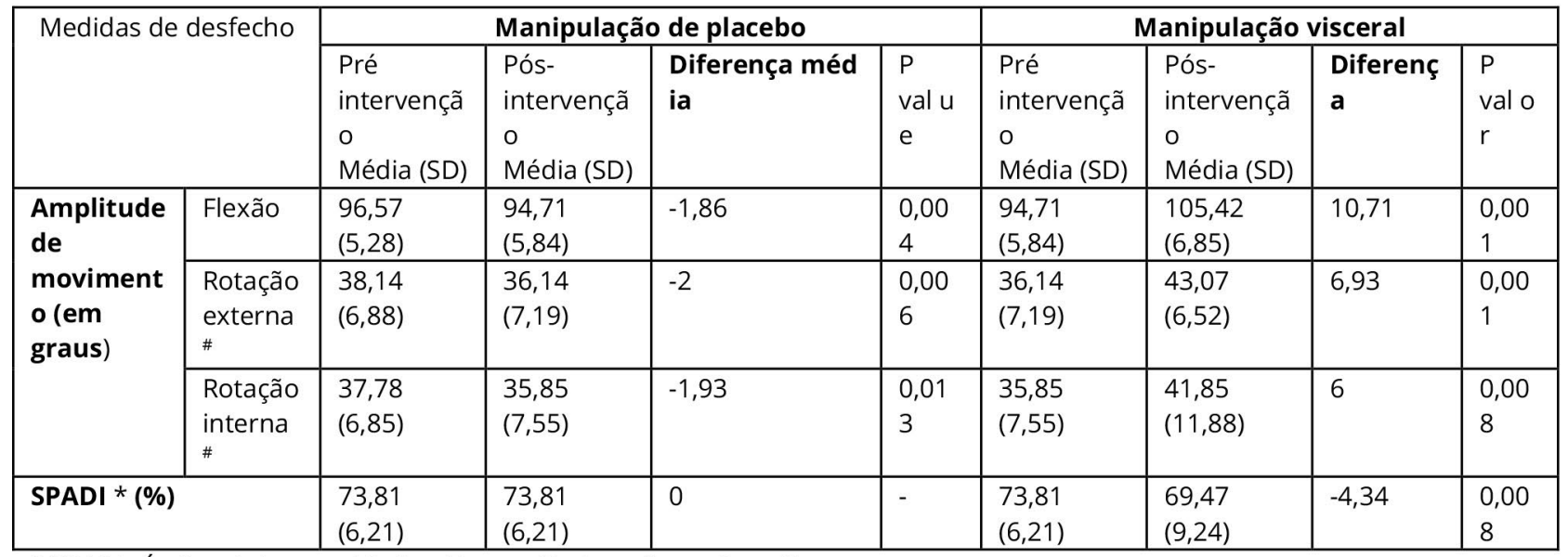

* SPADI - Índice de Incapacidade e Dor no Ombro , " rot - Rotação

Quadro 3. Mostrando dentro do grupo a comparação de todas as medidas de resultado de ambos os grupos.

\begin{tabular}{|l|l|l|l|l|l|l|l|l|}
\hline & \multicolumn{2}{|l|}{ Manipulação placebo } & \multicolumn{3}{l|}{ Manipulação visceral } \\
\hline $\begin{array}{l}\text { Força } \\
\text { (em } \\
\text { mm Hg) }\end{array}$ & $\begin{array}{l}\text { Pré } \\
\text { Intervenção }\end{array}$ & $\begin{array}{l}\text { Pós- } \\
\text { Intervenção }\end{array}$ & Diferença média & $\begin{array}{l}\text { VALOR } \\
\text { de P }\end{array}$ & $\begin{array}{l}\text { Pré } \\
\text { Intervenção }\end{array}$ & $\begin{array}{l}\text { Pós- } \\
\text { intervenção }\end{array}$ & $\begin{array}{l}\text { Significar } \\
\text { Diferença }\end{array}$ & $\begin{array}{l}\text { VALOR } \\
\text { de P }\end{array}$ \\
\hline Flexores & $\begin{array}{l}57,85 \\
(24,39)\end{array}$ & $\begin{array}{l}56,42 \\
(23,56)\end{array}$ & $-1,43$ & 0,335 & $\begin{array}{l}56,64 \\
(24,70)\end{array}$ & $\begin{array}{l}54,64 \\
(21,16)\end{array}$ & -2 & 0,54 \\
\hline $\begin{array}{l}\text { Rotação } \\
\text { externa }\end{array}$ & $\begin{array}{l}48,21 \\
(15,14)\end{array}$ & $\begin{array}{l}48,21 \\
(15,14)\end{array}$ & 0 & - & $\begin{array}{l}48,21 \\
(15,14)\end{array}$ & $\begin{array}{l}48,21 \\
(15,14)\end{array}$ & 0 & - \\
\hline $\begin{array}{l}\text { Rotação } \\
\text { interna }\end{array}$ & $\begin{array}{l}43,92 \\
(16,77)\end{array}$ & $\begin{array}{l}43,92 \\
(16,7)\end{array}$ & 0 & $\begin{array}{l}43,92 \\
(16,77)\end{array}$ & $\begin{array}{l}43,92 \\
(16,77)\end{array}$ & 0 & - \\
\hline
\end{tabular}

^rot -Músculos rotadores 
No grupo de manipulação placebo, a diferença média foi -1,86 para flexão, $-2,00$ para rotação externa e $-1,93$ para rotação interna. A diferença média foi -1,43 para a força dos músculos flexores. No grupo de manipulação visceral, a diferença média foi de 10,71 para flexão, de 6,93 para rotação externa, 6,00 para rotação interna e -4,34 para a diferença média do SPADI.

A comparação intra-grupo de ambos os grupos mostrou uma significância estatística em todos os três planos de movimentos e na pontuação do SPADI. A diferença média da força para flexores foi -1,43 no placebo e -2,00 no grupo manipulação visceral. Nas leituras pré e pós para força dos rotadores externos e internos não houve diferença entre os dois grupos.

A comparação intra-grupo de ambos os grupos para as medidas de desfecho de força não mostrou alterações estatisticamente significativas.

Quadro 4. Mostra entre a comparação do grupo de medidas de resultados

\begin{tabular}{|c|c|c|c|c|c|}
\hline \multirow{2}{*}{\multicolumn{2}{|c|}{ Medidas de desfecho }} & \multirow{3}{*}{$\begin{array}{l}\text { Diferença média } \\
8,86 \\
\end{array}$} & \multicolumn{2}{|c|}{ Intervalo de confiança } & \multirow{3}{*}{$\begin{array}{l}\text { Valor } \mathrm{F} \\
<0,001 \\
\end{array}$} \\
\hline & & & \multirow{2}{*}{$\begin{array}{l}\text { Limite inferior } \\
4,50 \\
\end{array}$} & \multirow{2}{*}{$\begin{array}{l}\text { Limite superior } \\
13,21 \\
\end{array}$} & \\
\hline Amplitude & Flexão & & & & \\
\hline $\begin{array}{l}\text { de } \\
\text { movimento }\end{array}$ & $\begin{array}{l}\text { Rotação } \\
\text { externa\# }\end{array}$ & 8,93 & 5,56 & 12,29 & $<0,001$ \\
\hline & $\begin{array}{l}\text { Rotação } \\
\text { interna \# }\end{array}$ & 7,93 & 3,73 & 12,12 & $<0,001$ \\
\hline \multirow[t]{3}{*}{ Força } & Flexores & $-0,57$ & $-7,84$ & 6,70 & 0,873 \\
\hline & 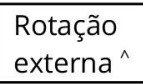 & 0 & - & - & - \\
\hline & $\begin{array}{l}\text { Rotação } \\
\text { interna }\end{array}$ & 0 & - & - & - \\
\hline \multicolumn{2}{|l|}{ SPADI $^{*}$} & 4,34 & $-7,19$ & $-1,48$ & 0,004 \\
\hline
\end{tabular}

*SPADI - Índice de Incapacidade e Dor no Ombro, " rot - Rotação, ^ rot - Rotadores

Neste estudo, a comparação entre os grupos mostrou uma melhora estatisticamente significativa no grupo de manipulação visceral para as medidas de desfecho, amplitude de movimentos de flexão, rotação externa e rotação interna e na pontuação SPADI, com valor de $p<0,001$ e 0,004 respectivamente. Não houve mudanças estatisticamente significativas na força dos flexores do ombro.

\section{Discussão}

O objetivo do estudo foi explorar o efeito da manipulação visceral na dor, incapacidade e mobilidade em indivíduos com capsulite adesiva do ombro direito. O resultado deste estudo mostrou uma melhora na mobilidade, na dor e na incapacidade após a manipulação visceral. Houve aumento da flexão, rotação externa e rotação interna do ombro direito medidos pelo goniômetro digital e notável melhora na atividade funcional do ombro e no escore de dor medido pelo escore (SPADI) após duas semanas de manipulação visceral.

A manipulação visceral tem demonstrado aumentar a comunicação proprioceptiva através da relação mecânica no interior do corpo, diminuindo assim o limiar de dor, a anormalidade estrutural e a postura defeituosa (manipulação visceral de Barral e manipulação neural do novo manual articular de Approach). O nervo frênico que inerva o ombro direito também inerva o fígado. Esta conexão anatômica entre o fígado e o ombro direito fornece a forte conexão e liberação de restrição dos movimentos da articulação do ombro e tensão muscular ao redor do ombro direito através do método direto e indireto de manipulação visceral, assim, evidenciando melhora na mobilidade, na dor e na incapacidade após a intervenção ${ }^{5}$. 
Resultado semelhante doi encontrado no estudo realizado por Silva e colaboradores sobre o efeito da manipulação visceral do estômago e do fígado na dor cervical. A amplitude de movimento cervical e a atividade do músculo trapézio mostrou que houve diminuição da dor e aumento da atividade do trapézio superior. As alterações trazidas pela manipulação visceral na amplitude de movimentos podem estar relacionadas ao mecanismo de liberação de tensão na membrana de suporte que conecta o sistema visceral e o musculoesquelético, resultando no aumento da comunicação proprioceptiva, consequentemente, aliviar os sintomas de dor e disfunção ${ }^{5}$.

A articulação glenoumeral é a articulação mais móvel do corpo, os movimentos da superfície articular do ombro dependem da atividade dos músculos circundantes. Estudos anteriores mostraram que qualquer atividade muscular anormal pode restringir os movimentos do ombro e levar à disfunção articular? Durante a reabilitação fisioterapêutica, é importante examinar os sintomas do paciente, o estágio da doença e os diferentes padrões de movimento. De acordo com Yang e colaboradores, o ritmo insuficiente escapuloumeral e a inclinação posterior da escápula durante a elevação do braço são importantes a se considerar na reabilitação do paciente com capsulite adesiva. As estratégias de tratamento para restaurar a mobilidade do ombro enfatizam a mobilização do ombro e a aplicação de tensão na cápsula, ligamentos extracapsulares e tendões musculares do manguito rotador, na tentativa de alongamento dos tecidos restritivos ${ }^{10}$.

De acordo com evidências recentes e diretrizes clínicas para o manejo da capsulite adesiva, o tratamento deve ser composto por injeção de esteróides (4-6weeks), conscientização do paciente, modalidades eletroterapêuticas, mobilizações articulares, manipulações e exercícios de fortalecimento. Os resultados estão de acordo com a afirmação de Gluraiz e colaboradores de que os fatores que geram dor são multidimensionais. A dor pode ser sentida como resultado de disfunções no sistema músculo-esquelético, nervoso, visceral, psicossomático e emocional do corpo ${ }^{11}$. Portanto, uma avaliação e intervenção multissistêmica abrangente será eficaz para aliviar a dor e melhorar a disfunção do movimento. A avaliação musculoesquelética integrada à avaliação visceral e sua manipulação é uma das ferramentas mais eficazes para as estratégias de reabilitação ${ }^{12}$.
Não houve melhora significativa observada na força muscular dos flexores de ombro, rotadores externos e rotadores internos, o que pode ser atribuído ao fato de o tônus muscular não ser influenciado pela manipulação visceral. Intervenções fisioterapêuticas com exercícios de fortalecimento terá um papel vital na ativação dos músculos após a manipulação visceral.

\section{Limitações}

As limitações deste estudo são o pequeno tamanho da amostra, o seguimento não foi avaliado para estudar a retenção do efeito do tratamento e uma medida de desfecho mais objetiva, como a eletromiografia, poderia ter fornecido uma visão mais profunda da atividade muscular do ombro. Um ensaio clínico randomizado controlado, com um tamanho de amostra maior e registro eletromiográfico da atividade muscular do ombro com acompanhamentos é recomendado para estudos futuros.

\section{Conclusão}

A manipulação visceral do fígado demonstrou aumentar a amplitude de movimentos do ombro direito (flexão, rotação externa e rotação interna) e diminuir a dor e a incapacidade funcional em indivíduos com capsulite adesiva do ombro direito. Trata-se de uma ferramenta eficaz em uma intervenção multi-dimensional para reabilitação da capsulite adesiva.

\section{Contribuições dos autores}

Ghillodia A e Gandhi BK conceberam a pesquisa, o desenho do estudo, coletaram e analisaram os dados. Ambos os autores aprovaram a versão final do manuscrito.

\section{Conflitos de interesses}

Nenhum conflito financeiro, legal ou político envolvendo terceiros (governo, empresas e fundações privadas, etc.) foi declarado para nenhum aspecto do trabalho submetido (incluindo, mas não se limitando a subvenções e financiamentos, participação em conselho consultivo, desenho de estudo, preparação de manuscrito, análise estatística, etc.). 


\section{Referências}

1. Page RS, McGee SL, Eng K, Brown G, Beattie S, Collier F et al. Adhesive capsulitis of the shoulder: protocol for the adhesive capsulitis biomarker (AdCaB) study. BMC musculoskeletal disorders. 2019;20(1):145. doi: 10.1186/s12891-019-2536-X

2. Satpathy M, Jain S, Gupta R, Agrawal S. Comparative evaluation of results of physiotherapy alone, periarticular injections followed by physical exercise and manipulation under anaesthesia followed by physical exercise in adhesive capsulitis of shoulder. Int J. Orthopaedics. 2019;5(4):147-151. doi: 10.22271/ortho. 2020. v6.i1c.1851

3. Dias R, Cutts S, Massoud S. Frozen shoulder. BMJ. 2005;331:1453. doi: 10.1136/bmj.331.7530.1453

4. Le HV, Lee SJ, Nazarian A, Rodriguez EK. Adhesive capsulitis of the shoulder: review of pathophysiology and current clinical treatments. Shoulder \& elbow. 2017;9(2):75-84. doi: $10.1177 / 1758573216676786$

5. Silva ACO, Biasotto-Gonzalez DA, Oliveira FHM, Andrade AO, Gomes CAFP, Lanza FC et al. Effect of osteopathic visceral manipulation on pain, cervical range of motion, and upper trapezius muscle activity in subjects with chronic nonspecific neck pain and functional dyspepsia: a randomized, doubleblind, placebo-controlled pilot study. Evidence-Based Complementary and Alternative Medicine. 2018;2018:4929271. doi: $10.1155 / 2018 / 4929271$

6. MacDermid JC, Solomon P, Prkachin K. The Shoulder Pain and Disability Index demonstrates factor, construct and longitudinal validity. BMC musculoskeletal disorders. 2006;7:12: doi:

10.1186/1471-2474-7-12
7. Correll S, Field J, Hutchinson H, Mickevicius G, Fitzsimmons A, Smoot B. Realibility And Validity Of The Halo Digital Goniometer For Shoulder Range Of Motion In Healthy Subjects. Int J Sports Phys Ther. 2018;13(4):707-714. doi: 10.26603/ijspt20180707

8. Bhinderwala S, Bedekar N. Reliability of modified sphygmomanometer for measurement of maximum isometric shoulder muscle strength. Physiotherapy-The Journal of Indian Association of Physiotherapists. J Indian Assoc Physiother. 2019;13(1):9-13. doi: 10.4103/PJIAP.PJIAP 1518

9. Rawat P, Eapen C, Seema KP. Effect of rotator cuff strengthening as an adjunct to standard care in subjects with adhesive capsulitis: A randomized controlled trial. J Hand Ther. 2017;30(3):235-241. doi: 10.1016/j.jht.2016.10.007

10. Yang JL, Chang CW, Chen SY, Lin JJ. Shoulder kinematic features using arm elevation and rotation tests for classifying patients with frozen shoulder syndrome who respond to physical therapy. Man Ther. 2008;13(6):544-51. doi: 10.1016/j. math.2007.07.006

11. Naqvi M, Shahid M, Nizami GN, Ali SH. Practice of Ergonomics among the bankers of Private and Public Sector Banks. Pak J Rehab. 2012;1(2):1-7.

12. Noten S, Meeus M, Stassijn G, Van Glabbeek F, Verborgt O, Struyf F. Efficacy of different types of mobilization techniques in patients with primary adhesive capsulitis of the shoulder: a systematic review. Arch Phys Med Rehabil. 2016;97(5):815-25. doi: 10.1016/j.apmr.2015.07.025 